\title{
ESTRATÉGIA EDUCATIVA VOLTADA PARA ENFERMEIROS SOBRE ATENÇÃO BÁSICA À INFERTILIDADE: ESTUDO DE INTERVENÇÃO
}

\author{
Andrezza Alves DIAS $S^{\mathrm{a}}$ Escolástica Rejane Ferreira MOURA ${ }^{\mathrm{b}}$, Paula Sacha Frota NOGUEIRAc, \\ Janaína Fonseca Victor COUTINHO ${ }^{d}$, Mônica Oliveira Batista ORIÁ
}

\section{RESUMO}

Objetivou-se avaliar o impacto de estratégia educativa abordando assistência à infertilidade e verificar práticas introduzidas no cotidiano laboral dos participantes após intervenção. Pesquisa-ação, desenvolvida com 11 enfermeiras da Estratégia Saúde da Família de Fortaleza-CE, por meio da leitura de fôlder em "roda de conversa". Os dados foram coletados em agosto e outubro de 2010, anteriormente, imediatamente após e decorridos 60 dias da intervenção. Constatou-se aumento do nível de conhecimento imediatamente após a intervenção, quando todas as participantes obtiveram nível de conhecimento "mais que bom" ou "muito bom”, deixando de se enquadrarem nos níveis "pouco" e "bom”, como ocorreu antes da intervenção; com 60 dias, registrou-se nível de conhecimento "bom”, constatando relativo declínio no conhecimento. Houve autorreconhecimento com relação à capacidade de introduzir práticas no cotidiano laboral após a intervenção, confirmando impacto positivo da intervenção.

Descritores: Enfermagem. Infertilidade. Conhecimento.

\section{RESUMEN}

El objetivo fue evaluar impacto de estrategia educativa dirigida a la asistencia a la infertilidad y verificar prácticas en el trabajo diario de los participantes después de esta intervención. Investigación-acción, desarrollada con 11 enfermeros de la Estrategia de Salud Familiar en Fortaleza-CE, mediante lectura del folleto en "rueda de conversación." Los datos fueron recolectados en agosto y octubre de 2010 , antes, inmediatamente después, y transcurridos 60 días de la intervención. Se comprobó aumento del nivel de conocimiento inmediatamente después de la intervención, cuando todos las participantes obtuvieron nivel de conocimiento "más que bueno" o "muy bueno", dejando los niveles de ajuste "poco" y "bueno" como antes de la intervención; con 60 días fue recolectado nivel de conocimiento "bueno", con disminución relativa en el conocimiento. Hubo autorreconocimiento de la capacidad de iniciar prácticas en la rutina laboral después de la intervención, lo que confirma el impacto positivo de esta.

Descriptores: Enfermería. Infertilidad. Conocimiento.

Título: Estrategia educativa para enfermeros en atención primaria a la infertilidad: estudio de intervención.

\section{ABSTRACT}

The objective was to assess the impact of an educational strategy approaching infertility assistance; and verify practices introduced into the daily work of the participants after this intervention. Research-action developed with 11 nurses from the Family Health Strategy, in Fortaleza-CE, through the reading of a brochure in a "circle of conversation." Data were collected in August and October 2010, before, immediately after and 60 days after the intervention. An increase in the levels of knowledge was noted immediately after the intervention, when all participants had a "more than good" or "very good" level of knowledge, leaving behind the "little" and "good" levels rated before the intervention; at 60 days, a "good" level of knowledge was noted again, showing a relative decrease in knowledge. There was self-recognition of the ability to introduce practices into the daily work after the intervention, confirming the positive impact thereof.

Descriptors: Nursing. Infertility. Knowledge.

Title: Educational strategy addressed to nurses in primary care for infertility: an intervention study.

a Enfermeira da UTI neonatal do Hospital Geral de Fortaleza, Fortaleza, Ceará, Brasil

b Doutora em Enfermagem, Professora Adjunto IV do Departamento de Enfermagem da Universidade Federal do Ceará (UFC), Fortaleza, Ceará, Brasil.

c Mestre em Enfermagem, Professora da Universidade de Fortaleza (UNIFOR), Fortaleza, Ceará, Brasil.

d Doutora em Enfermagem, Professora Adjunto I do Departamento de Enfermagem da UFC, Fortaleza, Ceará, Brasil.

e Doutora em Enfermagem, Professora Adjunta II do Departamento de Enfermagem da UFC, Fortaleza, Ceará, Brasil. 


\section{INTRODUÇÃO}

Infertilidade é a incapacidade de conceber após um ano de vida sexual ativa, com frequência de coito de pelo menos duas vezes por semana, sem uso de método anticoncepcional ${ }^{(1)}$.

Estima-se que, em todo o mundo, existam de 50 a 80 milhões de casais inférteis, ocorrendo cerca de dois milhões de novos casos por ano ${ }^{(2)}$. Esse evento é vivido por 8 a $15 \%$ dos casais em geral. No Brasil, mais de 278 mil casais têm dificuldade de gerar um filho em algum momento de sua idade fértil ${ }^{(3)}$. Portanto, a infertilidade constitui problema relevante de Saúde Pública, em que 70\% dos casos podem ser solucionados na atenção básica, com ações e procedimentos de baixo custo; os demais pacientes (30\%) teriam suas necessidades atendidas em serviços de referência de média e alta complexidade $^{(4)}$.

A atual Política Nacional de Atenção Integral à Saúde da Mulher (PNAISM), instituída em 2004, apresenta como um de seus objetivos específicos a ampliação e qualificação da atenção ao Planejamento Familiar (PF), incluindo a assistência à infertilidade ${ }^{(5)}$.

A partir da Portaria $\mathrm{N}^{\circ}$. 426, de 22 de março de 2005, foi instituída no âmbito do Sistema Único de Saúde (SUS), a Política Nacional de Atenção Integral em Reprodução Humana Assistida que, dentre outras resoluções, é responsável por identificar os determinantes e condicionantes dos principais problemas de infertilidade de casais em idade fértil e definir critérios técnicos para o funcionamento efetivo dos serviços de atenção básica, média e alta complexidade voltados ao tratamento da infertilidade. Estabeleceu-se, então, que a atenção básica deve ser responsável pela identificação do casal infértil, na qual devem ser realizados anamnese, exame clínico-ginecológico e um elenco de exames complementares de diagnósticos básicos, afastando-se patologias, fatores concomitantes e situações que interfiram em uma futura gestação e que ponha em risco a vida da mulher ou do feto ${ }^{(6)}$.

Já a Portaria $\mathrm{N}^{\circ}$. 388, de 06 de julho de 2005, da Secretaria de Atenção à Saúde, foi instituída para complementar a portaria anteriormente citada, determinando que na atenção básica devem-se realizar três consultas médicas e duas de enfermagem, e serem solicitados exames de colpocitologia oncótica, sorologia anti-Vírus da Imunodeficiência Humana (HIV), hepatites B e C, sífilis (VDRL), toxoplasmose
IgG, glicemia de jejum e espermograma, além da vacinação contra a rubéola ${ }^{(7)}$.

Apesar do que está determinado nas referidas Portarias, o atendimento oferecido por algumas equipes da Estratégia Saúde da Família (ESF), responsáveis pela atenção básica no país, encontra-se aquém do que está preconizado. Em 72,9\% dos municípios brasileiros, a atenção à infertilidade, que deveria estar integrada ao conjunto de ações desenvolvidas na atenção ao PF, não é realizada ${ }^{(8)}$. Estudo realizado em Florianópolis-SC, utilizando o método de análise do Discurso do Sujeito Coletivo, com nove enfermeiros que atuam na ESF, afirmou não ser oferecido atendimento aos casos de infertilidade na atenção básica, sendo estes logo encaminhados para médico especialista (ginecologista) e, em seguida, referenciados para serviços de média ou alta complexidade (ambulatório ou hospital), perdendo-se o vínculo com a Unidade Básica de Saúde (UBS), por não haver contra-referência ${ }^{(9)}$.

Estudo realizado no Sistema de Saúde de Fortaleza-CE, com 171 enfermeiros integrantes da ESF após analisar o conhecimento e prática destes acerca do conceito de infertilidade, tipos e causas, bem como as ações assistenciais passíveis de serem desenvolvidas neste nível da atenção, concluiu haver déficit de conhecimento dos enfermeiros e que a atenção aos casais inférteis é ausente ou inadequada $^{(10)}$. Portanto, a realidade encontrada em Fortaleza-CE foi semelhante à de Florianópolis-SC, em que ambas se caracterizaram pela fragilidade da atenção em infertilidade na ESF.

Experiência de sucesso no acompanhamento de casais inférteis vem sendo desenvolvida na Clínica de Educação para Saúde, da Universidade do Sagrado Coração de Bauru-SP, na qual 16 casais foram acompanhados e receberam orientações sobre o Método de Billingss ou da Ovulação. Destes, nove já conseguiram engravidar e três bebês já nasceram. Geralmente, o casal consegue a gravidez dois anos após a adoção do método. Alguns já haviam sido encaminhados para bancos de sêmen e para fertilização in vitro, porém conseguiram engravidar apenas com adoção dos métodos baseados na percepção da fertilidade ${ }^{(11)}$. Apesar da reduzida amostra, seus resultados reforçam a possibilidade de resolubilidade da atenção básica aos casos de infertilidade, porém somente estudos mais abrangentes permitirão fazer inferências. É necessário que existam profissionais capacitados para atender a esta população e serviços organizados para oferecer suporte. 
Em face ao exposto, percebe-se que o atendimento às pessoas com necessidades no campo da infertilidade nas UBS é, muitas vezes, ausente ou de baixa resolubilidade, repercutindo, por vezes, em encaminhamentos desnecessários à média e alta complexidade. Entretanto, a realização de consultas de Enfermagem e de consultas médicas por profissionais capacitados para introduzir atividades de aconselhamento aos casais, oferecer orientações para concentrarem as relações sexuais no período fértil e que eliminem qualquer fator que interfira no depósito do sêmen ou que dificulte a migração espermática através do trato genital feminino (lubrificantes, duchas vaginais, preservativos, diafragma, espermicidas, dispositivo intra-uterino (DIU), coito interrompido e outros), conforme recomenda a Portaria $\mathrm{N}^{\circ} .388$, poderá modificar este quadro $^{(7)}$. Nesse contexto, questionou-se: a aplicação de uma estratégia educativa sobre assistência à infertilidade pode melhorar o conhecimento e a prática de enfermeiros que atuam na ESF, nessa área do cuidado? Para responder a tal inquietação, definiu-se pela realização do presente estudo com os seguintes objetivos: avaliar o impacto de estratégia educativa abordando assistência à infertilidade para enfermeiros da ESF; e verificar ações práticas introduzidas no cotidiano laboral dos participantes após a intervenção.

\section{METODOLOGIA}

Pesquisa-ação, desenvolvida na Secretaria Executiva Regional (SER) I do município de Fortaleza-CE. O município está dividido em seis SER, modelo de organização administrativo idealizado para facilitar a gestão local em suas várias faces, incluindo o setor saúde ${ }^{(12)}$. A SER I foi escolhida devido à conveniência de deslocamento para autora que realizou o trabalho de campo. Esta possui 38 enfermeiros na ESF, distribuídos nas 13 UBS. Todos foram convidados para participar do estudo, porém 11 compareceram.

A pesquisa-ação consiste em planejar - agir - descrever - avaliar, e proporciona uma interação entre pesquisador e sujeito pesquisado ${ }^{(13)}$. Assim, a estratégia educativa consistiu na leitura dirigida de um folder, elaborado por todas as autoras, em "roda de conversa", metodologia adaptada da "roda de gestão". Este é um método que aumenta a capacidade de análise e intervenção por parte dos participantes a partir da escuta e interpretação coletiva do sujeito, além de criar uma circulação de afeto e confiança entre os membros ${ }^{(14)}$. Ocorreram duas rodas de conversa, em agosto de 2010, com duração aproximada de 50 minutos cada, com cinco e seis enfermeiras, respectivamente.

No primeiro momento foi aplicado um formulário contendo perguntas sobre idade, sexo, instituição de graduação, tempo de graduação, tempo de atuação em $\mathrm{PF}$, tempo de atuação em ESF e sobre participação em curso sobre PF que incluísse atenção básica à infertilidade, de modo a caracterizar as participantes.

As participantes foram avaliadas antes da estratégia educativa por meio de um questionário contendo 12 perguntas de múltipla escolha, com apenas uma resposta correta, que versou sobre conceito e tipos de infertilidade, fatores relacionados à infertilidade, anamnese e exame físico feminino e masculino e ações passíveis de serem realizadas nesse nível de atenção. Imediatamente após, as mesmas foram reavaliadas com o mesmo questionário e em seguida a pesquisadora comentou as questões e esclareceu dúvidas. Por fim, após 60 dias da intervenção, além de reavaliação feita com o mesmo questionário, as participantes foram investigadas sobre a aplicação prática da formação, ou seja, o impacto nas atividades laborais cotidianas, que foi verificado pela checagem de ações desenvolvidas pelas participantes.

Portanto, para avaliar se a intervenção educativa modificou a prática dos enfermeiros com relação à atenção básica à infertilidade, definiu-se o intervalo de dois meses como adequado, porém não invalida que tal avaliação seja repetida em intervalos futuros. Deve-se reconhecer, entretanto, que o tempo decorrido da formação poderá atenuar o desempenho profissional com relação a determinado treinamento recebido, em particular quando faltam condições para aplicação das mudanças e supervisão apoiadora.

Os dados referentes ao conhecimento foram analisados conforme somatório das 12 questões, em que cada questão respondida corretamente valeu 0,8 ponto, podendo o nível de conhecimento de cada participante variar de "nenhum conhecimento" a "muito bom conhecimento", conforme a nota obtida (quadro 1). A escala de avaliação de conhecimento adotada neste estudo, apresentada no referido quadro, foi utilizada em pesquisa realizada com 40 pacientes para determinar o nível de conhecimento sobre a gestão dos fatores de risco relacionados à 


\begin{tabular}{|l|l|}
\hline Nível de conhecimento & Nota \\
\hline Nenhum conhecimento & 0 \\
\hline Muito pouco conhecimento & $\geq 1-2,9$ \\
\hline Pouco conhecimento & $3-4,9$ \\
\hline Bom conhecimento & $5-6,9$ \\
\hline Mais que bom conhecimento & $7-8,9$ \\
\hline Muito bom conhecimento & $9-10,0$ \\
\hline
\end{tabular}

Quadro 1 - Escala de avaliação do nível de conhecimento. Fonte: Zernike e Henderson, 1998.

hipertensão, antes e após a intervenção com duas estratégias de ensino ${ }^{(15)}$.

Os dados coletados nos três momentos foram organizados em tabelas e gráficos e a análise comparativa foi realizada, na perspectiva de identificar o impacto sobre o conhecimento e a prática dos enfermeiros no atendimento de pessoas com infertilidade antes da estratégia educativa, imediatamente após e com 60 dias.

O projeto de pesquisa foi submetido ao Comitê de Ética da Universidade Federal do Ceará (UFC), obtendo parecer favorável por meio do protocolo $n^{\circ}$ 172/10. Foram atendidas as exigências da Resolução 196/96, do Conselho Nacional de Saúde, que trata das questões éticas da pesquisa envolvendo seres humanos ${ }^{(16)}$. As participantes foram informadas sobre os objetivos da pesquisa e assinaram o Termo de Consentimento Livre e Esclarecido.

\section{RESULTADOS E DISCUSSÃO}

Todas as participantes foram do sexo feminino, característica histórica da Enfermagem, pois esta nasceu como um serviço organizado de ordens religiosas que impuseram, por longo período, o exercício institucional majoritariamente feminino e caritativo; consideraram-se, ainda, a influência de Florence Nightingale que institucionalizou na Inglaterra Vitoriana (1862), uma profissão para as mulheres, na qual estas eram "naturalmente preparadas" a partir de valores que se consideravam femininos, como o cuidar, por exemplo ${ }^{(17)}$.

A idade variou entre 28 e 48 anos, com uma média de 35,36 anos e desvio padrão de 6,54. O tempo de graduação variou de 4 a 23 anos, com média de 10,54 e desvio padrão de 5,50. O tempo de atuação em PF variou de um mês a 15 anos, sendo semelhante ao de atuação na ESF que ficou entre dois meses e 14 anos, apresentando médias de 4,04 e 3,91, respectivamente. Portanto, as participantes constituíram um grupo heterogêneo com relação às variáveis levantadas, aspecto positivo ao estudo, uma vez que os resultados versarão sobre profissionais de maior e menor tempo de atuação, mais ou menos experientes na área pesquisada.

Todas as participantes graduaram-se em uma das três principais instituições de ensino superior do Estado do Ceará, sendo 5 egressas da Universidade Estadual do Ceará, 3 da Universidade Federal do Ceará e outras 3 da Universidade de Fortaleza.

Somente 3 enfermeiras referiram ter participado de curso sobre PF, porém nenhum abordou atenção básica à infertilidade. As capacitações oferecidas para estes profissionais se limitaram à contracepção, contribuindo para que a prática voltada aos casais inférteis esteja ausente ou inadequada, o que restringe o direito constitucional do cidadão de receber atenção integral à saúde reprodutiva ${ }^{(5)}$.

A tabela 1 apresentada a seguir mostra os níveis de conhecimentos das enfermeiras nos três momentos de avaliação do estudo.

$\mathrm{Na}$ avaliação anterior à intervenção as questões que abordaram conhecimento sobre anamnese, exame físico e métodos baseados na percepção da fertilidade foram as que obtiveram maior porcentagem de acertos. As questões sobre exame físico da mulher (questão 6) e orientação adequada do método de Billings ou muco-cervical (questão 10), tiveram acerto pelas 11 participantes. Saber orientar sobre o método de Billings é crucial na atenção em concepção.

A questão 1 apresentou maior índice de erro (10 enfermeiras), pois estas deixaram de levar em conta a frequência de relações sexuais semanais. Sabe-se que, segundo o Ministério da Saúde, é infértil o casal que após um ano de vida sexual ativa, com frequência de coito de pelo menos duas vezes por semana e sem uso de método anticoncepcional, não conseguir engravidar ${ }^{(1)}$. Desconhecer esse conceito pode resultar em falta de incentivo à frequência de coitos semanais e ao maior insucesso nos resultados, bem como gerar diagnósticos de infertilidade falsos positivos, que irão desencadear expectativas indevidas aos usuários.

Os tipos de infertilidade (questão 2) e ações e/ou procedimentos que devem ser oferecidos pela equipe de ESF (questão 12), foram, cada uma, assinaladas corretamente por quatro enfermeiras, demonstrando pouco conhecimento do grupo. 
Tabela 1 - Distribuição do número de enfermeiras que apresentaram respostas corretas por questão na avaliação anterior à intervenção, imediatamente após e 60 dias após. Secretaria Executiva Regional I - SER I. Fortaleza-Ceará, ago-out., 2010.

Questões avaliadas $(n=11)$

Conhecimento sobre infertilidade, tipos e causas

1 Conceito de infertilidade

2 Tipos de infertilidade

3 Fatores relacionados à infertilidade feminina

4. Fatores relacionados à infertilidade masculina

Conhecimento sobre anamnese, exame físico e métodos baseados na percepção da fertilidade

5 Anamnese nos casos de infertilidade

6 Exame físico de mulheres com queixa de infertilidade

7 Exame físico de homens com queixa de infertilidade

8 Métodos baseados na percepção da fertilidade

9 Orientações sobre temperatura basal

10 Orientações sobre método de Billings

ou muco-cervical

11 Orientações sobre método Ogino-Knaus ou tabela

Conhecimento de ações passíveis de serem realizadas na atenção básica

12 Conjunto de ações e/ou procedimentos que devem ser oferecidos pela equipe de ESF

Nível de conhecimento das participantes

Nenhum

Muito pouco

Pouco

Bom

Mais que bom

Muito bom

$\begin{array}{lll}\text { Avaliação } & \text { Avaliação } & \text { Avaliação após } \\ \text { anterior à } & \text { imediatamente } & 60 \text { dias da } \\ \text { intervenção } & \text { após a intervenção } & \text { intervenção }\end{array}$

1

4

8

9

7

11

7

8

7

11

8

4

8

$\begin{array}{lll}- & - & - \\ - & - & - \\ 4 & - & - \\ 3 & - & 4 \\ 4 & 8 & 6 \\ - & 3 & 1\end{array}$

Em relação aos tipos de infertilidade, os itens assinalados incorretamente foram "natural, cirúrgica e idiopática”, por quatro participantes, e "masculina, feminina e mista", por três. Sabe-se que os tipos de infertilidade são dois: a primária, quando o casal nunca concebeu, e a secundária, quando o casal já possui um ou mais conceptos. Já em relação ao desconhecimento das ações que devem ser realiza- das na atenção básica, há prejuízo aos usuários que necessitam de atendimento voltado à infertilidade, pois os mesmos acabam sendo referenciados ao nível secundário ou terciário, enfrentando longas e demoradas filas de espera, sobrecarregando, assim, o sistema.

$\mathrm{Na}$ avaliação imediatamente após a intervenção, oito enfermeiras apresentaram nível de 


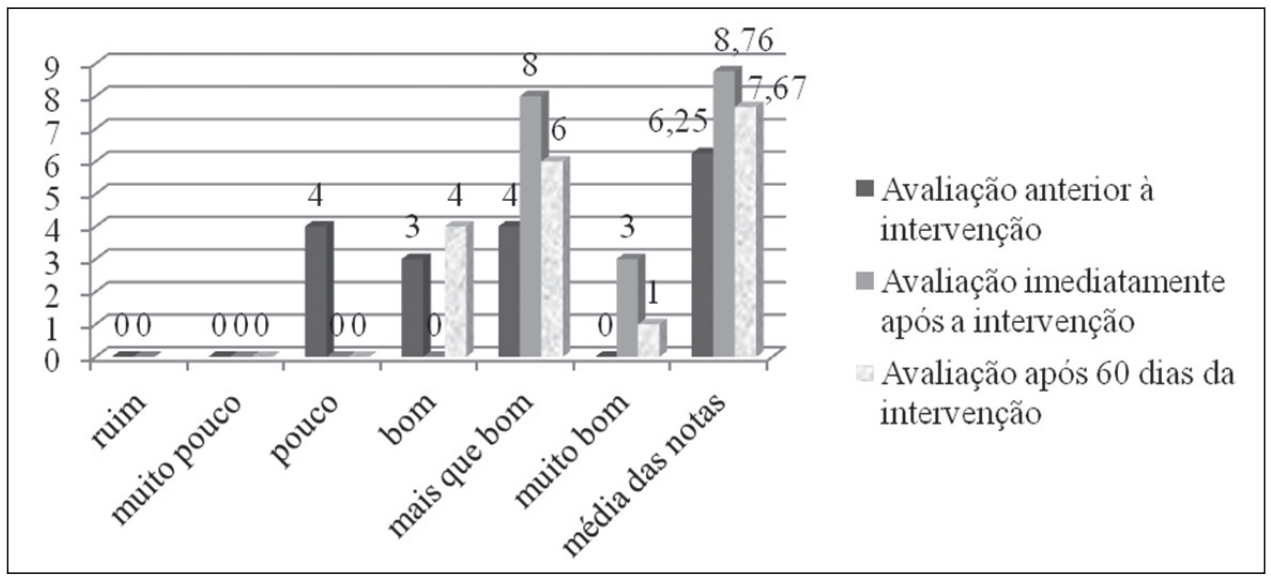

Gráfico 1 - Distribuição das enfermeiras segundo o nível de conhecimento nas três avaliações e média das notas obtidas. Unidades de Saúde da SER I. Fortaleza-Ceará, ago-out, 2010.

conhecimento "mais que bom" e três "muito bom", ou seja, um aumento expressivo. Esse achado vem ao encontro do que foi constatado em estudo quase-experimental, prospectivo e comparativo cujo objetivo foi avaliar duas estratégias educativas para a prevenção do diabetes mellitus tipo 2 em adolescentes com fatores de risco, divididos no grupo A $(n=45)$, que utilizou como estratégia educativa $o$ folder com explicações breves, e no grupo $B(n=45)$, um programa educativo de cinco encontros, com duração total de dez horas. Este concluiu que as estratégias educativas adotadas mostraram-se eficazes para aumentar o conhecimento acerca dos temas propostos $(\mathrm{p}=0,0001)$, imediatamente após a intervenção ${ }^{(18)}$.

Quando comparadas as avaliações antes e imediatamente após a intervenção, a melhora no nível de conhecimento se deu nas questões sobre conceito de infertilidade, tipos e causas; e sobre ações passíveis de serem realizadas na atenção básica. As questões sobre anamnese (questão 5), métodos baseados na percepção da fertilidade (questão 8), orientação sobre o método de Billings ou muco-cervical (questão 10) e sobre o método Ogino-Knaus ou tabela, foram acertadas por todas as participantes, demonstrando um maior conhecimento acerca da abordagem inicial do casal infértil e quais os métodos indicados para o reconhecimento do período fértil. Nas demais questões o número máximo de erro foi três, que ocorreu nas questões sobre exame físico do homem (questão 7) e as ações que devem ser desenvolvidas nas equipes da ESF (questão 12). As participantes referiram que não é comum a presença masculina no atendimento realizado na atenção básica. Tal fato pode justificar o conhecimento reduzido acerca do exame físico masculino, pois, como não há demanda, logo, não há prática, e sem praticar, o conhecimento tende a diminuir.

$\mathrm{Na}$ avaliação 60 dias após as questões que obtiveram totalidade de acerto foram: os fatores relacionados à infertilidade feminina (questão 3) e sobre o método de Billings ou muco-cervical (questão 10). O conceito de infertilidade (questão 1) obteve o maior índice de erro - por sete participantes, pelas mesmas percepções manifestas na primeira avaliação.

O gráfico 1 oferece melhor visualização dos resultados das três avaliações, bem como as médias das notas obtidas pelas participantes, favorecendo uma análise mais comparativa.

Observa-se no gráfico 1 aumento do nível de conhecimento imediatamente após a estratégia educativa, em que todas as participantes obtiveram nível de conhecimento "mais que bom" ou "muito bom", deixando de se enquadrarem nos níveis "pouco" e "bom", como apareceu na avaliação anterior à intervenção. Já na avaliação após 60 dias, o nível de conhecimento "bom" voltou a ser detectado, constatando relativo declínio no conhecimento adquirido.

Em relação às médias obtidas nas notas, a elevação prosseguida de declínio é esperada, pois a avaliação de conhecimento pós-formação guarda relação com a memorização do conteúdo por parte dos sujeitos, o que por sua vez depende do tipo de apresentação e/ou método de ensino/aprendizagem adotado. A roda de conversa (técnica participativa) constitui método de elevado 
Tabela 2 - Distribuição do número de enfermeiras de acordo com as ações que se sentiram aptas a realizar após a estratégia educativa. Unidades de Saúde da SER I. Fortaleza-Ceará, out, 2010.

\begin{tabular}{lc}
\hline Variáveis (n=10) & $\mathbf{N}^{\mathbf{0}}$ \\
\hline Ações que se sentiram aptos a realizar & 6 \\
Anamnese & 5 \\
Realização de exame físico & 7 \\
Orientação quanto aos métodos de percepção da fertilidade & 5 \\
Orientação quanto às barreiras à fecundação que podem ser evitadas & 3 \\
Realização de exames complementares & 7 \\
Encaminhamento para consulta médica & 1 \\
Outros (a estratégia educativa ampliou e corrigiu conhecimentos prévios sobre infertilidade &
\end{tabular}

percentual de memorização de conteúdo (cerca de $80 \%$ ), justificando o aumento da média das notas imediatamente após a intervenção ${ }^{(19)}$. Já introduzir ações no cotidiano profissional não está relacionado somente às estratégias de educação utilizadas para tal, também depende de tempo, disposição e possibilidades para que as mudanças venham a ocorrer (recursos, ambiente) $)^{(18)}$.

Quanto a avaliação após 60 dias, dois participantes referiram mudanças em sua prática profissional; um negou; cinco afirmaram mudança em parte e três afirmaram que já realizavam ações compatíveis com a estratégia educativa. Estes resultados correspondem a uma auto-avaliação das enfermeiras, ou seja, as mesmas afirmam uma intenção ou motivação para uma nova prática profissional, uma vez que não foi possível detectar atendimento a casos de infertilidade no período de 60 dias após a estratégia educativa. Esse resultado guarda relação com a rotina de algumas unidades de saúde, cuja clientela com essa necessidade é encaminhada ao médico, sem passar pela consulta de Enfermagem. Ademais, é baixa a procura por este serviço, uma vez que é raramente ofertado, levando a clientela a procurar serviço de média ou alta complexidade mediante manifestação de infertilidade. A esse respeito, enfermeiros e equipe da ESF que têm como uma de suas atribuições cuidar de indivíduos inférteis devem planejar-se para oferecer atendimento nessa área ${ }^{(20)}$.

A tabela 2 sintetiza as ações passíveis de serem ofertadas na atenção básica por parte do grupo estudado.

As ações preconizadas pelo Ministério da Saúde, conforme as Portarias $\mathrm{N}^{\mathrm{o}} 426$ e $^{\mathrm{o}} 388$ foram apresentadas em seis itens, conforme apresentados na tabela $2^{(6-7)}$. Somente a participante que referiu não haver mudança em sua prática profissional, não assinalou nenhum dos itens da tabela 2 , justificando $\mathrm{o} \mathrm{n}=10$. As demais assinalaram um ou mais itens, conforme se sentissem aptas a realizá-los, justificando a soma total maior que o $\mathrm{n}$.

Houve auto-reconhecimento por parte das enfermeiras com relação à capacidade de introduzir ações práticas no cotidiano laboral após a intervenção, pois agora estas conhecem e/ou sentem-se aptas a realizarem as ações preconizadas pelo Ministério da Saúde. Assim, espera-se que o atendimento às pessoas com necessidade no campo da infertilidade torne-se uma realidade no cenário deste estudo.

\section{CONSIDERAÇÕES FINAIS}

A intervenção educativa mostrou melhoria no nível de conhecimento das enfermeiras quanto à atenção em infertilidade. A média das notas obtidas na avaliação anterior à intervenção foi 6,25, aumentando para 8,76 imediatamente após e diminuindo para 7,67 no último momento.

Apesar de a avaliação quanto a ações introduzidas na prática laboral ter sido prejudicada pela ausência de clientela nas UBS de atuação das enfermeiras ao longo dos 60 dias, sete enfermeiras apresentaram intenção ou motivação para uma nova prática, se auto-avaliando como aptas para introduzirem ações preconizadas nas Portarias $\mathrm{N}^{\circ}$ $426 \mathrm{e} \mathrm{N}^{\circ} 388$.

Propõe-se que nas ações de planejamento familiar a atenção à infertilidade seja divulgada. Isso pode 
ganhar força por meio da divulgação em cartazes, panfletos, visitas domiciliárias e no setor de marcação de consultas, informando à clientela sobre o atendimento.

Sugere-se para pesquisas futuras, a ampliação desta estratégia educativa para as demais SER do município de Fortaleza, bem como para outros municípios e regiões.

\section{REFERÊNCIAS}

1 Ministério da Saúde (BR), Assistência em Planejamento Familiar: Manual Técnico. $4^{\mathrm{a}}$. ed. Brasília (DF): Editora MS, 2002.

2 Gonçalves, J. Avaliação do casal infértil. Rev Port Clin Geral. 2005; 21:493-503.

3 Ministério da Saúde(BR). Média e alta complexidade: média complexidade reprodução humana assistida [Internet]. Brasília (DF), 2009. [citado 2010 mar 25]. Disponível em: http://portal.saude.gov.br/ portal/sas/mac/area.cfm?id_area $=832$.

4 Pedrosa, J. Política prevê atenção integral. Jornal de Brasília [Internet]. 30 jun 2005. [citado $2010 \mathrm{abr}$ 29]. Disponível em: http://www.sistemas.aids.gov. $\mathrm{br} /$ imprensa/Noticias.asp?NOTCod $=65742$.

5 Ministério da Saúde (BR), Política Nacional de Atenção à Mulher: Princípios e Diretrizes [Internet]. Brasília (DF), 2004. [citado 2010 abr 10]. Disponível em: http://bvsms.saude.gov.br/bvs/publicacoes/ politica_nac_atencao_mulher.pdf.

6 Ministério da Saúde (BR), Portaria No 426/GM, de 22 de março de 2005 [Internet]. Brasília (DF), 2005. [citado $2010 \mathrm{abr} 16$ ]. Disponível em: http:// dtr2001.saude.gov.br/sas/PORTARIAS/Port2005/ GM/GM-426.htm.

7 Ministério da Saúde (BR), Portaria No 388, de 06 de julho de 2005 [Internet]. Brasília (DF), 2005. [citado 2010 abr 16]. Disponível em: http://docs.google.com/ gview? $\mathrm{a}=\mathrm{v} \& \mathrm{q}=$ cache\%3AcpTLVfgbDG4J\%3Awww. saude.mg.gov.br\%2Fatos_normativos\%2Flegislacao-sanitaria\%2Festabelecimentos-de-saude $\% 2$ Fatencao-em-reproducao-humana-assistida- $1 \% 2$ F Portaria_388.pdf + Portaria $+\mathrm{N} \% \mathrm{C} 2 \% \mathrm{BA}+388 \% 2 \mathrm{C}+\mathrm{de}+\mathrm{O}$ $6+$ de + julho + de $+2005 \&$ hl $=$ pt $-B R \&$ sig $=$ AFQjCNEz hd7TSTf60wSucth2zXZOG5Bgjw\&pli=1.

8 Costa AM, Guilhem D, Silver LD. Planejamento familiar: a autonomia das mulheres sob questão. Rev Bras Saude Mater Infant. 2006; 6(1):75-84.
9 Lindner SR, Coelho EBS, Büchele F, Soares C. Direitos reprodutivos: o discurso e a prática dos enfermeiros sobre planejamento familiar. Cogitare Enferm. 2006; 11(3): 197-205.

10 Moura, ERF, Vieira RPR, Dias AA. Atenção básica a infertilidade na perspectiva de enfermeiros da Estratégia Saúde da Família. Relatório de pesquisa-Edital PIBIC/CNPq 2009/2010, Universidade Federal do Ceará. Fortaleza: UFCE; 2010.

11 Universidade do Sagrado Coração; Clínica de Educação para a Saúde. Método promove controle natural da fertilidade [Internet]. 2009. [citado 2010 abr 22]. Disponível em: http://www.providafamilia.org.br./ site/index.php.

12 Junqueira LAP, Inojosa RM. Experiências Municipais: desenvolvimento social e intersetorialidade na gestão pública municipal. Conferência Nacional de Saúde [Internet]. 2010. [citado 2010 maio 12]. Disponível em: http://www.datasus.gov.br/cns/ inovador/FORTALEZA.htm.

13 Monteiro CFS, Moreira MRC, Oliveira EAR, Moura MES, Costa JV. Pesquisa-ação: contribuição para prática investigativa do enfermeiro. Rev Gaúcha Enferm. 2010; 31(1):167-174.

14 Campos GWS. Um método para análise e co-gestão de coletivos: a constituição do sujeito, a produção do valor de uso e a democracia em instituições: o método da roda. São Paulo: Hucitec; 2000.

15 Zernike W, Henderson A. Evaluating the effectiveness of two teaching strategies for patients diagnosed with hypertension. J Clin Nurs; v.7, p.37-44, 1998.

16 Ministério da Saúde (BR), Conselho Nacional de Saúde, Comitê Nacional de Ética em Pesquisa em Seres Humanos. Resolução 196, de 10 de outubro de 1996: diretrizes e normas regulamentadoras de pesquisa envolvendo seres humanos. Brasília (DF); 2003.

17 Lopes MJM, Leal SMC. A feminização persistente na qualificação profissional da enfermagem brasileira. Cad Pagu. 2005;24:105-125.

18 Silva ARV. Avaliação de duas estratégias educativas para a preservação do diabetes mellitus tipo $2 \mathrm{em}$ adolescentes [tese]. Fortaleza: Programa de Pós-Graduação em Enfermagem, Universidade Federal do Ceará; 2009. 
19 Dale E. Cone of experience. In: Merrill C. Educational Media: Theory into Practice. Columbus, Ohio: Wiman RV, 1969.
20 Taspinar A, Kavlak O, Sirin A. Differences in quality of life between infertile women and men in Turkey. J Obst Gynecol Neon Nurs.2010; 39(2):191-198.

Endereço do autor / Dirección del autor / Author's address:

Andrezza Alves Dias

Av. Sargento Hermínio Sampaio, 1415, ap. 1204B, São Gerardo 60320-105, Fortaleza, CE

E-mail: andrezzaalvesdias@,hotmail.com 